\title{
Vitamin D status and COVID-19 in older adults
}

\author{
Amit K. J. Mandal ${ }^{1}$ - Vadir Baktash ${ }^{1} \cdot$ Tom Hosack $^{1} \cdot$ Constantinos G. Missouris ${ }^{1,2}$
}

Received: 5 September 2020 / Accepted: 11 September 2020 / Published online: 21 September 2020

(c) Springer Nature Switzerland AG 2020

We read with interest the evidence presented by Daneshkhah et al. [1] for a potential relationship between vitamin D deficiency and unregulated cytokine release leading to complications in COVID-19 patients. Their analysis of mean 25-OH-D levels of older adults in countries with similar testing strategies may suggest a role of vitamin $\mathrm{D}$ in reducing the adaptive average of time-adjusted case mortality ratio. A significant limitation, perhaps, is the use of historical vitamin D levels in their meta-analysis.

Our recently published prospective cohort study in the United Kingdom assessed the importance of vitamin D deficiency in older patients with COVID-19 [2]. Although our study was underpowered to detect a mortality difference between deficient and replete groups, the significant findings were that patients with lower concentrations of $25 \mathrm{OH}-\mathrm{D}$ $(\leq 30 \mathrm{nmol} / \mathrm{l})$ at the time of acute infection demonstrated clinically relevant, elevated markers of cytokine release syndrome and were more likely to become hypoxic and require ventilatory support. Our data compliment emerging evidence that vitamin D status may be a surrogate prognosticator for morbidity and mortality [3].

The relationship between vitamin D deficiency and adverse prognosis has been suggested by mortality and hospitalisation rates for COVID-19 seen to be higher in northern latitude countries compared to those closer to the equator [4]. Institutionalised older adults are inherently at risk of vitamin D deficiency as a result of a lack of sun exposure and dietary insufficiency and may have worse outcomes with COVID-19. There is also growing concern that the Black, Asian and Minority Ethnic (BAME) community, who produce less vitamin $\mathrm{D}$ as a result of higher skin melanin

Constantinos G. Missouris

dinos.missouris@nhs.net

1 Departments of Cardiology and Internal Medicine, Wexham Park Hospital, Frimley Health NHS Foundation Trust, Wexham Street, Slough, UK

2 University of Cyprus Medical School, Nicosia, Cyprus content, are more susceptible to severe presentations of COVID-19.

It is established that there is a complex interplay between vitamin $\mathrm{D}$ and various components of the innate (enhancement) and adaptive (inhibition) immune responses to both bacterial and viral infections. In addition to anti-inflammatory properties, vitamin $\mathrm{D}$ exerts a protective effect on alveolar epithelial cells, preserves endothelial integrity and reduces vascular permeability. Vitamin D also induces expression of ACE-2. While increased ACE-2 expression was initially predicted to amplify infection risk and severity, paradoxically, ACE-2 has also been shown to protect against acute lung injury [5]. Disruption of any of these defensive mechanisms may explain the association between vitamin D deficiency and requirement of ventilatory support in our cohort-whether this represents a causal relationship is unclear.

Regardless of the aetiology, vitamin D deficiency is readably diagnosable and correctable. On the basis of this reasoning and pending results of trials evaluating vitamin $\mathrm{D}$ supplementation and appropriate dosing within the context of COVID-19, efforts to achieve reference nutrient intakes of vitamin $\mathrm{D}$ according to national guidelines should be encouraged.

Funding The authors received no specific funding for this work.

\section{Compliance with ethical standards}

Conflict of interest No potential conflict of interest was reported by the authors.

Ethical approval This survey (2) was approved by the trust audit department with reference FH119 and with clinically collected, non-identifiable data which does not fall under the remit of NHS Research Ethics Committee. All data was collected locally and handled in accordance with European General Data Protection Regulation (GDPR) standards, as well as local and NHS standards on data protection. 
Informed consent All practices conducted as part of this study were done in accordance with local regulations and best clinical practice protocols. Serum vitamin D levels were tested alongside the hospital routine serum COVID-19 panel and did not require any extra phlebotomy. Patients received care in line with standard practices for the management of COVID-19 infection throughout the study period.

\section{References}

1. Daneshkhah A, Agrawal V, Eshein A et al (2020) Evidence for possible association of vitamin D status with cytokine storm and unregulated inflammation in COVID-19 patients. Aging Clin Exp Res. https://doi.org/10.1007/s40520-020-01677-y
2. Baktash V, Hosack T, Patel N et al (2020) Vitamin D status and outcomes for hospitalised older patients with COVID-19. Postgrad Med J. https://doi.org/10.1136/postgradmedj-2020-138712

3. Munshi R, Hussein MH, Toraih EA et al (2020) Vitamin D insufficiency as a potential culprit in critical COVID-19 patients. J Med Virol. https://doi.org/10.1002/jmv.26360

4. Panarese A, Shahini E (2020) Covid-19 and vitamin D. Aliment Pharm Ther 51:993-995. https://doi.org/10.1111/apt.15752

5. Kuka K, Imai Y, Penninger JM (2006) Angiotensin-converting enzyme 2 in lung diseases. Curr Opin Pharmacol 6:271-276

Publisher's Note Springer Nature remains neutral with regard to jurisdictional claims in published maps and institutional affiliations. 